\title{
Diversidad, abundancia y distribución de peces en el río Bulubulu
}

\section{Diversity, abundance and distribution of fishes in Bulubulu river}

\author{
Holbach Núñez ${ }^{1^{*}} \&$ Antonio Torres² \\ ${ }^{1}$ Consultor independiente \\ ${ }^{2}$ Docente-investigador, Universidad de Guayaquil, Facultad de Ciencias Naturales, \\ Laboratorio de acuacultura
}

Recibido 10 de abril 2021; recibido en forma revisada 2 de mayo 2021, aceptado 4 de junio 2021 Disponible en línea 26 de junio 2021

\begin{abstract}
Resumen
Se realizó un trabajo en el río Bulubulu ubicado en la provincia del Guayas, con el objetivo de obtener un registro de la diversidad, abundancia y distribución de la ictiofauna presente en diez estaciones, utilizando dos artes de pesca. Como resultado se capturaron 428 individuos pertenecientes a cinco ordenes, nueve familias, 13 géneros, y 14 especies. Las especies más abundantes fueron Astyanax festae y Brycon dentex con 90 individuos cada una, seguidos de Brycon alburnus con 84 individuos, la especies más raras o escasas en el lugar fueron Astroblepus sp. Y Chaetostoma fischeri con un individuo cada una. Las especies con mayor distribución fueron A. festae y $B$. dentex, encontrándose en 7 estaciones cada una. En cuanto a la diversidad, el área de muestreo obtuvo un índice de Shannon de 2,03 (diversidad media), y las estaciones con valores altos fueron solamente 1 y 2 , con valores de 1,73 y 1,6 respectivamente.
\end{abstract}

Palabras claves: ictiofauna, distribución, diversidad, abundancia, índice de Shannon

\begin{abstract}
A study was done on the Bulubulu river located in the province of Guayas, with the aim of obtaining a record of the diversity, abundance and distribution of the ichthyofauna present in ten stations, using two fishing gear. As a result, 428 individuals were captured, belonging to five orders, nine families, 13 genera, and 14 species. The most abundant species were Astyanax festae and Brycon dentex with 90 individuals each, followed by Brycon alburnus with 84 individuals, the rarest species in the place were Astroblepus sp. And Chaetostoma fischeri with one individual each. The species with greater distribution were $A$. festae and $B$. dentex, being in 7 stations each. Regarding diversity, the sampling area obtained a Shannon index of 2.03 (average diversity), and stations with high values were only 1 and 2 , with values of 1.73 and 1.6 respectively.
\end{abstract}

Keywords: ichthyofauna, distribution, diversity, abundance, Shannon index.

\section{Introducción}

Los cuerpos hídricos poseen una alta biodiversidad de especies animales y vegetales, alcanzando una importancia ecológica, económica e inclusive cultural (Tognelli, Lasso, Bota-Sierra, JiménezSegura y Cox, 2016). De esta riqueza faunística, los peces representan el grupo más abundante; Se han reportado aproximadamente 28000 especies de peces entre marinos $(58 \%)$, de agua dulce $(41 \%)$ y estuarinas en un 1\% (Helfman, Collette, y Facey, 1997; Nelson, 2006; Leveque, Oberdorff, Paugy, Stiassny, Tedesco, 2008).

En términos regionales, Sudamérica cuenta con 4475 especies de peces y probablemente existan 1550 no descritas, representando una alta biodiversidad en la región tropical (Reis et al., 2003).

Ecuador posee una amplia diversidad de peces que habitan en aguas continentales y muchas de estas ellas son cultivadas como especies comerciales y de subsistencia; por lo tanto, los cuerpos hídricos en Ecuador presentan importancia socioeconómica para las comunidades asentadas en su entorno (López et al., 2015).

Un aspecto generalizado es que, en Ecuador existe carencia de un registro oficial de los peces de aguas continentales. Aquello puede conllevar a un mal manejo del recurso, poniendo en peligro la perpetuidad de las especies. Entre los ríos que han

* Correspondencia del autor:

E-mail: holb_stev94@hotmail.com 
sido objeto de investigación se encuentran el Guayas, Esmeraldas, Napo y Pastaza; mientras que, los ríos Morona Santiago, Zamora y Upano localizados en el oriente ecuatoriano, poseen escasa o nula información acerca de la ictiofauna presente (Anaguano, 2013).

El río Bulubulu, ubicado en las provincias de Guayas y Cañar, es un cuerpo de agua utilizado con distintos propósitos, una de las actividades principales es la pesca artesanal, ya sea de subsistencia o comercial (El telégrafo, 2016). Sin embargo, no existen estudios sobre la ictiofauna presente en esta cuenca, lo que conlleva a un pobre conocimiento asociado a las especies existentes su abundancia y su variación a lo largo de la cuenca.

Existe la posibilidad de encontrar diferencias en la estructura comunitaria de peces en diferentes zonas del rio Bulubulu, por lo tanto, el objetivo del presente estudio es realizar un análisis referente a la diversidad, abundancia y distribución de la ictiofauna presente en el río Bulubulu.

\section{Antecedentes}

Uno de los primeros artículos publicados sobre la riqueza ictiofaunística del Ecuador fue realizado por James Bohlke (1958), a manera de informe, titulado "Estudio sobre peces de la familia Characidae del Ecuador", en la cual se registró al menos 50 especies de carácidos para toda la región.

Ovchynnick (1967) elaboró la primera lista oficial de peces continentales del Ecuador, en ella se reportó un total de 276 especies. Un año después (1968) publicó otra lista, en la cual se registró 295 especies. Finalmente, el mismo autor (1971) elaboró una tercera lista de peces continentales, en la cual se incluyó aspectos biológicos de cada especie, a diferencia de las listas anteriores, y en la cual registró un total de 306 especies.

En lo que se refiere a taxonomía de peces de agua dulce, Stewart y Barriga (1987), publicaron un estudio titulado "Ictiofauna del Río Napo", en este trabajo se registró alrededor de 473 especies y 225 géneros, estableciendo una diversidad ictiofaunística mayor a las reportadas para cualquier rio con tamaño similar en el mundo.

Barriga (1991), elaboró su lista de peces continentales del Ecuador, en ella se reportaron 708 especies correspondientes a 307 géneros y 61 familias. Luego, (1994), llevó a cabo un estudio en la región noroccidente del país, enfocándose en los ríos San Juan, Mira, Mataje y Esmeraldas, aquí se reportaron 34 familias y 82 especies. Dichos estudios sumaron 400 nuevos registros al listado ya conocido en ese momento de la ictiofauna para el Ecuador; esto incluye principalmente los resultados de los estudios del nororiente y noroccidente del país.

Massay (2009) realizó un estudio en la cuenca baja del rio Taura, aquí se registraron 121 individuos, colectando 25 especies de las cuales 4 fueron nuevos registros para aguas ecuatorianas.
Barriga (2012), en su trabajo titulado "Lista de peces de agua dulce e Intermareales del Ecuador” presentó una lista de ictiofauna más actualizada con su distribución, aquí se identificó 944 especies de peces de agua dulce, demostrando así la diversidad íctica que posee el país.

Laaz \& Torres (2014), publicaron el estudio "lista de peces continentales de la cuenca del río Guayas", con la finalidad de poder actualizar los registros en este lugar, como resultado se obtuvo 125 especies, estas se encontraron distribuidas en 14 órdenes y 41 familias, cabe destacar que del total de las especies registradas, 55 fueron estrictamente de agua dulce, las cuales corresponden al $45 \%$ del total de especies capturadas en el estudio.

Aucapiña (2017), en su trabajo de titulación realizó un inventario ictiológico del Río Ruidoso, ubicado en la provincia del Guayas, donde identificó 19 especies, distribuidas en 5 órdenes, 12 familias y 17 géneros, de las cuales una especie era introducida (Oreochromis sp), siendo el orden Characiformes el más representativo, y las especies Astyanax festae, Rhoadsia altipina y Bryconamericus brevirostris, las más abundantes en el área de estudio. El mismo año Mawyin (2017), generó otro trabajo de titulación denominado diversidad y abundancia ictiofaunística en el río culebra, ubicado en la misma provincia, donde se encontraron nueve especies, pertenecientes a tres órdenes y cinco familias. Clasificándose en seis especies nativas, dos endémicas y una introducida.

\section{Materiales y métodos}

\section{Área de estudio}

El río Bulubulu está ubicado en el cantón El Triunfo a $61 \mathrm{Km}$ de Guayaquil, situado en la parte suroeste de la provincia del Guayas. Limita al norte con los cantones Yaguachi, Marcelino Maridueña, al sur con el cantón Naranjal y la provincia de Cañar y al oeste con los cantones Yaguachi y Naranja (Fig.1)

Los muestreos en este lugar fueron realizados durante los meses de enero a diciembre del 2019. Para este propósito se establecieron 10 estaciones de muestreo con sus respectivas coordenadas, abarcando un total de $66 \mathrm{~km}$ de recorrido en la cuenca del río.

Las condiciones ambientales en el río Bulubulu fluctúan dependiendo de la zona que atraviese, por este motivo existen variaciones en la temperatura de sus aguas (Varela y Ron, 2018). En sus aguas se realizan actividades como turismo, hay zonas en que el río es utilizado para el riego de zonas agrícolas, y algunas partes del mismo se usan para la pesca.

\section{Artes de pesca utilizados Pesca de arrastre}

Se empleó una red de chinchorro que es una red de encierro, formada de malla rectangular, de 5 metros de ancho por 1,5 metros de alto, con un ojo de malla de $1 \mathrm{~cm}$ (Fig.2).

\section{Atarraya}

Para este método se utilizó una red circular en forma de sombrilla, con una línea de plomos en los bordes, con un diámetro de 5 metros, con luz de malla de 1 cm (Fig.3). 


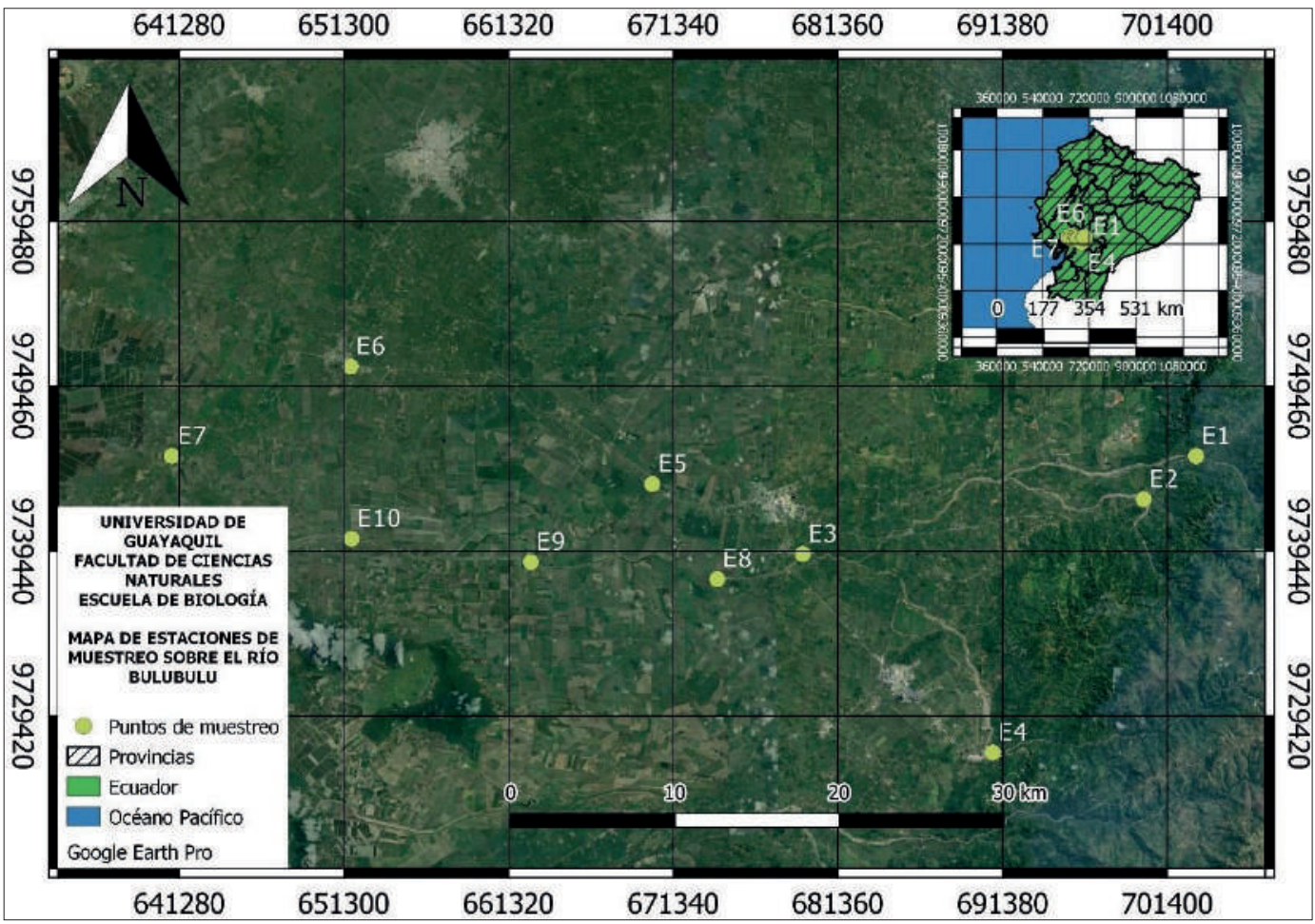

Figura 1. Estaciones de muestreo en el rio Bulubulu, Provincia del Guayas

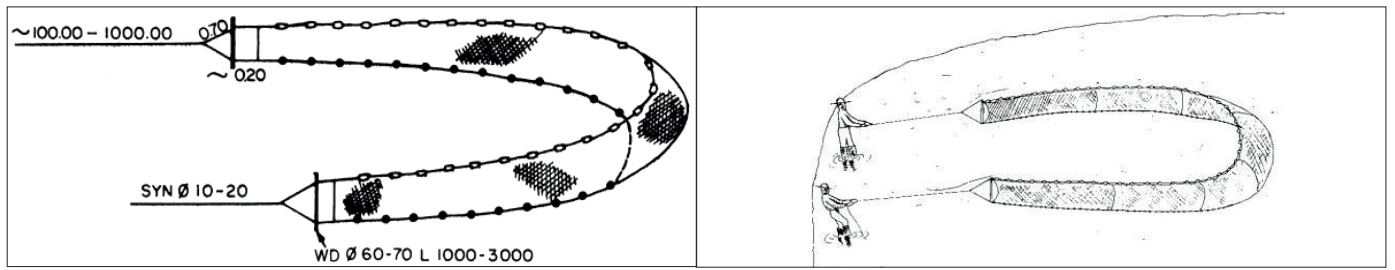

Figura 2. Esquema de la red de arrastre o Chinchorro: A) Forma de la red; B) Uso de red.

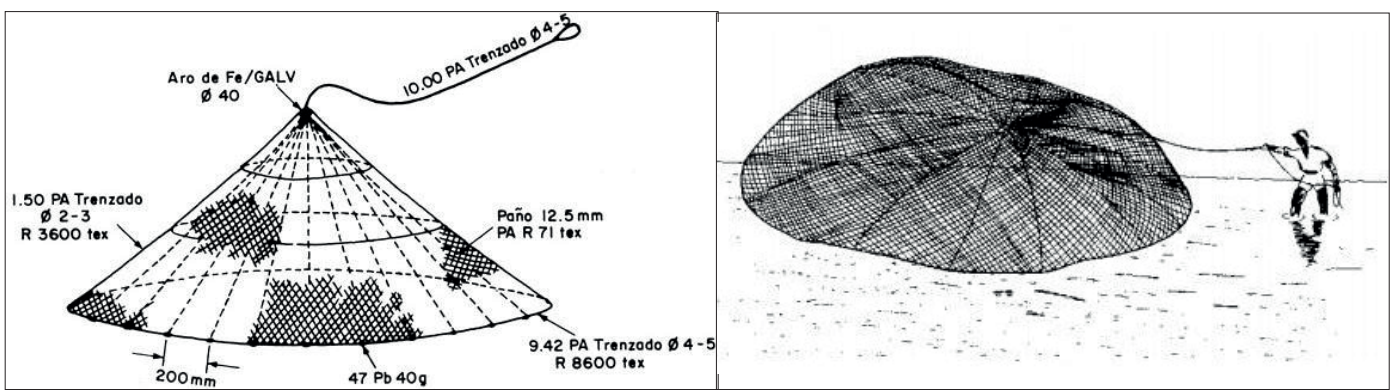

Figura 3. Esquema de la red de atarraya: A) forma de la red; B) Uso de la red

Preservación y transporte de muestras

Las muestras fueron fotografiadas y etiquetadas, anotando el sitio de muestreo, fecha de colecta, colector, posterior a esto, posteriormente se trasladaron al laboratorio LIRBA de la Facultad de Ciencias Naturales de la Universidad de Guayaquil, para este fin, los peces capturados fueron colocados en fundas y baldes que contenían una solución de formaldehído al 10\%. Las muestras se mantuvieron entre 24 a 48 horas en esta solución, posteriormente, fueron lavadas para ser colocadas en una solución de etanol al $70 \%$. 
Rev. Cient. Cien. Nat. Ambien. 15(1):211-227 Junio 2021
Núñez \& Torres - Diversidad, abundancia y distribución de peces en el río Bulubulu
Tabla 1. Índice de diversidad de Shannon para cada estación de muestreo

\begin{tabular}{cc}
\hline Estación & Índice de diversidad \\
\hline 1 & 1,728 \\
2 & 1,604 \\
3 & 1,333 \\
4 & 1,333 \\
5 & 1,256 \\
6 & 1,396 \\
7 & 1,046 \\
8 & 1,255 \\
9 & 1,456 \\
10 & 1,28 \\
\hline total & 2,029 \\
\hline
\end{tabular}

Análisis de datos

Identificación taxonómica de las especies

Se realizó con claves taxonómicas actualizadas
(Jiménez et al., 2014); (Maldonado et al., 2005); (Anzueto et al., 2013) y la Guía de (Laaz y Torres, 2014).

\section{Abundancia relativa}

Para determinar la abundancia relativa se realizó un conteo de número de individuos por especie, para determinar la abundancia total, y posteriormente por punto de muestreo.

\section{Diversidad}

Se calculó por medio del índice de Shannon-Weaver, para esto se usó el programa PAST. Que se basa en la formula a continuación.

$$
\left(H^{\prime}\right)=-(\text { pi ln pi). }
$$

Donde $\mathrm{pi}=\mathrm{ni} / \mathrm{N}$ y $\mathbf{N}=\mathbf{\sum n i} \mathrm{pi}$ : Abundancia relativa $\circ$ proporción de cada especie

ni: Número de individuos de la especie i

$\mathrm{N}$ : Población total

\section{Resultados}

En el área de estudio se encontraron 428 individuos, pertenecientes a cinco ordenes, nueve familias, 13

Tabla 2. Lista de especies encontradas en cada estación de muestreo del Río Bulubulu.

\begin{tabular}{|c|c|c|c|c|c|c|c|c|c|c|c|}
\hline ESPECIE & & & & & & & & & & & TOTAL \\
\hline & E1 & E2 & E3 & E4 & E5 & E6 & E7 & E8 & E9 & E10 & \\
\hline Achirus scutum & & & & & & & & & 3 & & 3 \\
\hline Andinoacara rivulatus & 1 & 6 & & & & 6 & & & & & 13 \\
\hline Astroblepus sp. & & 1 & & & & & & & & & 1 \\
\hline Astyanax festae & & & 19 & 3 & 18 & 19 & 10 & 10 & 11 & & 90 \\
\hline Brycon alburnus & 6 & 25 & & 18 & & 8 & 19 & 8 & & & 84 \\
\hline Brycon dentex & & & 17 & & 16 & 17 & 23 & 2 & 6 & 9 & 90 \\
\hline Chaetostoma fischeri & 1 & & & & & & & & & & 1 \\
\hline Eretmobrycon brevirostris & & & & & 1 & & & & & 5 & 6 \\
\hline Oreochromis sp. & & & 21 & 7 & 5 & & & 5 & 14 & 16 & 68 \\
\hline Pimelodella modestus & & & & & & & & & 4 & & 4 \\
\hline Poecilia reticulata & 5 & 10 & 8 & 3 & 3 & & & & & 6 & 35 \\
\hline Rhoadsia altipinna & 1 & 12 & & 4 & & 2 & & & & & 19 \\
\hline Transancistrus santarosensis & 4 & 3 & & & & & & & & & 7 \\
\hline Trychomicterus taenia & 3 & 4 & & & & & & & & & 7 \\
\hline TOTAL & 21 & 61 & 65 & 35 & 43 & 52 & 52 & 25 & 38 & 36 & 428 \\
\hline
\end{tabular}




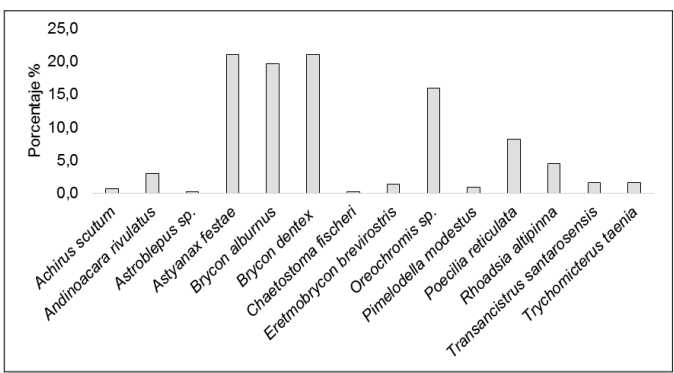

Figura 4. Abundancia relativa de peces en el río Bulubulu.

géneros, y 14 especies, de las cuales, las especies más abundantes fueron Astyanax festae y Brycon dentex, con un total de 90 individuos cada una, seguidos de Brycon alburnus con 84 individuos, la especies más raras o escasas en el lugar fueron Astroblepus sp. y Chaetostoma Fischeri con un total de un individuo cada especie (tabla 2 ).

\section{Diversidad}

Para todo el lugar de estudio se obtuvo un índice de diversidad de Shannon de 2,03 lo que indica una diversidad media en el lugar. Sin embargo en los resultados por estación se obtuvieron índices de diversidad bajos, exceptuando las estaciones 1 y 2 , las cuales mostraron valores de 1,72 y 1,6 respectivamente (Tabla 1 ).

\section{Abundancia}

Brycon dentex y Astyanax festae fueron las especies más abundante, seguidos de Brycon alburnus y Oreochromis sp. Por último Astroblepus sp. y Chaetostoma fischeri fueron las especies menos abundantes (Fig. 4).

\section{Resultados cuantitativos por estaciones de muestreo}

\section{Estación 1}

En este punto se lograron capturar 21 individuos, pertenecientes a cuatro órdenes, seis familias y siete géneros.

\section{Abundancia.}

Se puede apreciar que las especies más abundante fueron Brycon alburnus, Poecilia reticulata y Transancistrus santarosensis. Rhoadsia altipina, Andinoacara rivulatus y Chaetostoma fischeri fueron las menos abundantes (Tabla 3).

\section{Estación 2}

En este punto de muestreo se identificaron 61 individuos, pertenecientes a cuatro órdenes, siete familias y siete géneros.

\section{Abundancia}

Las especies más abundantes en este punto fueron Brycon alburnus, seguida de Rhoadsia altipina y Poecilia reticulata, la especie menos abundante fue Astroblepus sp. (Tabla 4).

\section{Estación 3}

En la estación 3, se capturaron 65 individuos, distribuidos en tres órdenes, cuatro familias y cuatro géneros.

\section{Abundancia.}

Las especies más abundantes fueron Oreochromis sp, Astyanax festae y Brycon dentex, la especie menos abundante fue Poecilia reticulata (Tabla 5).

\section{Estación 4}

En este punto de muestreo se encontraron 35 individuos, distribuidos en tres órdenes, cuatro familias y cinco géneros.

\section{Abundancia.}

Las especies más abundantes fueron Brycon alburnus y Oreochromis sp., las especies Astyanax festae y Poecilia reticulata fueron las menos abundantes (Tabla 6).

\section{Estación 5}

En este punto de muestreo se encontraron 43 individuos, distribuidos en tres órdenes, cuatro familias y cinco géneros.

Tabla 3. Composición y estructura de la ictiofauna presente en la estación 1 .

\begin{tabular}{ccccc}
\hline & & Estación 1 & & \\
\hline Orden & Familia & N. científico & N. de & $\begin{array}{c}\text { Porcentaje } \\
\%\end{array}$ \\
\hline Characiformes & Bryconidae & Brycon alburnus & 6 & 28,6 \\
Characiformes & Characidae & Rhoadsia altipina & 1 & 4,8 \\
Perciformes & Cichilidae & Andinoacara rivulatus & 1 & 4,8 \\
Siluriformes & Loricariidae & Chaetostoma fischeri & 1 & 4,8 \\
Siluriformes & Loricariidae & Transancistrus santarosensis & 4 & 19,0 \\
Siluriformes & Trychomicteridae & Trychomicterus taenia & 3 & 14,3 \\
Cyprinodontiformes & Poeciliidae & Poecilia reticulata & 5 & 23,8 \\
\hline & TOTAL & & 21 & 100 \\
\hline
\end{tabular}


Tabla 4. Composición y estructura de la ictiofauna presente en la estación 2.

\begin{tabular}{lllcc}
\hline \multicolumn{1}{c}{ Orden } & \multicolumn{2}{c}{ Estación 2 } & & \\
& Familia & N. científico & N. de & $\begin{array}{c}\text { Porcentaje } \\
\%\end{array}$ \\
\hline Characiformes & Bryconidae & Brycon alburnus & individuos & 41,0 \\
Characiformes & Characidae & Rhoadsia altipinna & 12 & 19,7 \\
Perciformes & Cichilidae & Andinoacara rivulatus & 6 & 9,8 \\
Siluriformes & Loricariidae & Transancistrus santarosensis & 3 & 4,9 \\
Siluriformes & Trychomicteridae & Trychomicterus taenia & 4 & 6,6 \\
Cyprinodontiformes & Poeciliidae & Poecilia reticulata & 10 & 16,4 \\
Siluriformes & Astroblepidae & Astroblepus sp. & 1 & 1,6 \\
\hline & & & 61 & 100,0 \\
\hline
\end{tabular}

Tabla 5. Composición y estructura de la ictiofauna presente en la estación 3.

\begin{tabular}{|c|c|c|c|c|}
\hline \multicolumn{5}{|c|}{ Estación 3} \\
\hline Orden & Familia & N. científico & $\begin{array}{c}\text { N. de } \\
\text { individuos }\end{array}$ & $\begin{array}{c}\text { Porcentaje } \\
\%\end{array}$ \\
\hline Cyprinodontiformes & Poeciliidae & Poecilia reticulata & 8 & 12,3 \\
\hline Characiformes & Characidae & Astyanax festae & 19 & 29,2 \\
\hline Characiformes & Bryconidae & Brycon dentex & 17 & 26,2 \\
\hline \multirow[t]{2}{*}{ Perciformes } & Cichilidae & Oreochromis sp. & 21 & 32,3 \\
\hline & TOTAL & & 65 & 100,0 \\
\hline
\end{tabular}

Tabla 6. Composición y estructura de la ictiofauna presente en la estación 4.

\begin{tabular}{ccccc}
\hline \multicolumn{5}{c}{ Estación 4 } \\
\hline Orden & Familia & N. científico & $\begin{array}{c}\text { N. de } \\
\text { individuos }\end{array}$ & $\begin{array}{c}\text { Porcentaje } \\
\%\end{array}$ \\
\hline Characiformes & Bryconidae & Brycon alburnus & 18 & 51,4 \\
Characiformes & Characidae & Rhoadsia altipinna & 4 & 11,4 \\
Perciformes & Cichilidae & Oreochromis sp. & 7 & 20,0 \\
Characiformes & Characidae & Astyanax festae & 3 & 8,6 \\
Cyprinodontiformes & Poeciliidae & Poecilia reticulata & 3 & 8,6 \\
\hline & TOTAL & & 35 & 100,0 \\
\hline
\end{tabular}

Tabla 7. Composición y estructura de la ictiofauna presente en la estación 5.

\begin{tabular}{ccccc}
\hline & \multicolumn{4}{c}{ Estación 5 } \\
\hline orden & Familia & N. científico & $\begin{array}{c}\text { N. de } \\
\text { individuos }\end{array}$ & Porcentaje \% \\
Characiformes & Characidae & Astyanax festae & 18 & 41,9 \\
Characiformes & Bryconidae & Brycon dentex & 16 & 37,2 \\
Cyprinodontiformes & Poeciliidae & Poecilia reticulata & 3 & 7,0 \\
Perciformes & Cichilidae & Oreochromis sp. & 5 & 11,6 \\
Characiformes & Characidae & Eretmobrycon brevirostris & 1 & 2,3 \\
\hline & TOTAL & & 43 & 100,0 \\
\hline
\end{tabular}




\section{Abundancia.}

Las especies más abundantes en esta estación fueron Astyanax festae y Brycon dentex, la especie menos abundante fue Eretmobrycon brevirostris. (Tabla 7)

\section{Estación 6}

En este punto de muestreo se capturaron 52 individuos, pertenecientes a dos órdenes, tres familias y cuatro géneros, y cinco especies.

\section{Abundancia}

Astyanax festae y Brycon dentex fueron las especies más abundantes, la especie menos abudante fue Rhoadsia altipina (Tabla 8).

\section{Estación 7}

En este punto se capturaron 52 individuos, pertenecientes a un orden, dos familias y tres géneros.

\section{Abundancia}

La especie con mayor abundancia fueron Brycon dentex y Brycon alburnus, La especie menos abudante fue Astyanax festae (Tabla 9).

\section{Estación 8}

En este punto de muestreo se capturaron 25 individuos, distribuidos en dos órdenes, tres familias, tres géneros y cuatro especies.

\section{Abundancia}

Las especies más abundantes fueron Astyanax festae y Brycon alburnus, la especie menos abundante fue Brycon dentex (Tabla 10).

\section{Estación 9}

En este lugar se encontraron 38 individuos pertenecientes a cuatro órdenes, cinco familias y cinco géneros.

\section{Abundancia}

Las especies con mayor abundancia fueron Oreochromis sp. y Astyanax festae, la especie menos abundante fue Achirus scutum (Tabla 11).

\section{Estación 10}

En este punto se capturaron 36 individuos, distribuidos en tres órdenes, cuatro familias y cuatro géneros.

\section{Abundancia.}

Las especies más abundantes en esta estación de muestreo fueron Oreochromis sp. y Brycon dentex, la especie menos abudnate fue Eretmobrycon brevirostris (Tabla 12).

\section{Distribución de especies}

Las especies que tuvieron distribución alta en las diferentes estaciones de muestreo fueron Astyanax festae y Brycon dentex, ambas estuvieron presentes en siete estaciones de muestreo (Figs. 8 y 10); Las especies con una distribución media fueron Brycon alburnus, Oreochromis sp. y Poecilla reticulata, estando presentes en un total de seis estaciones cada una (Figs. 9, 13 y 15). Por último, las especies con una distribución restringida fueron Achirus scutum, Astroblepus sp., Chaetostoma fischeri y Pimelodella modestus, las cuales estuvieron presentes en solamente una estación cada una (Figs. 5, 7, 11, 14).

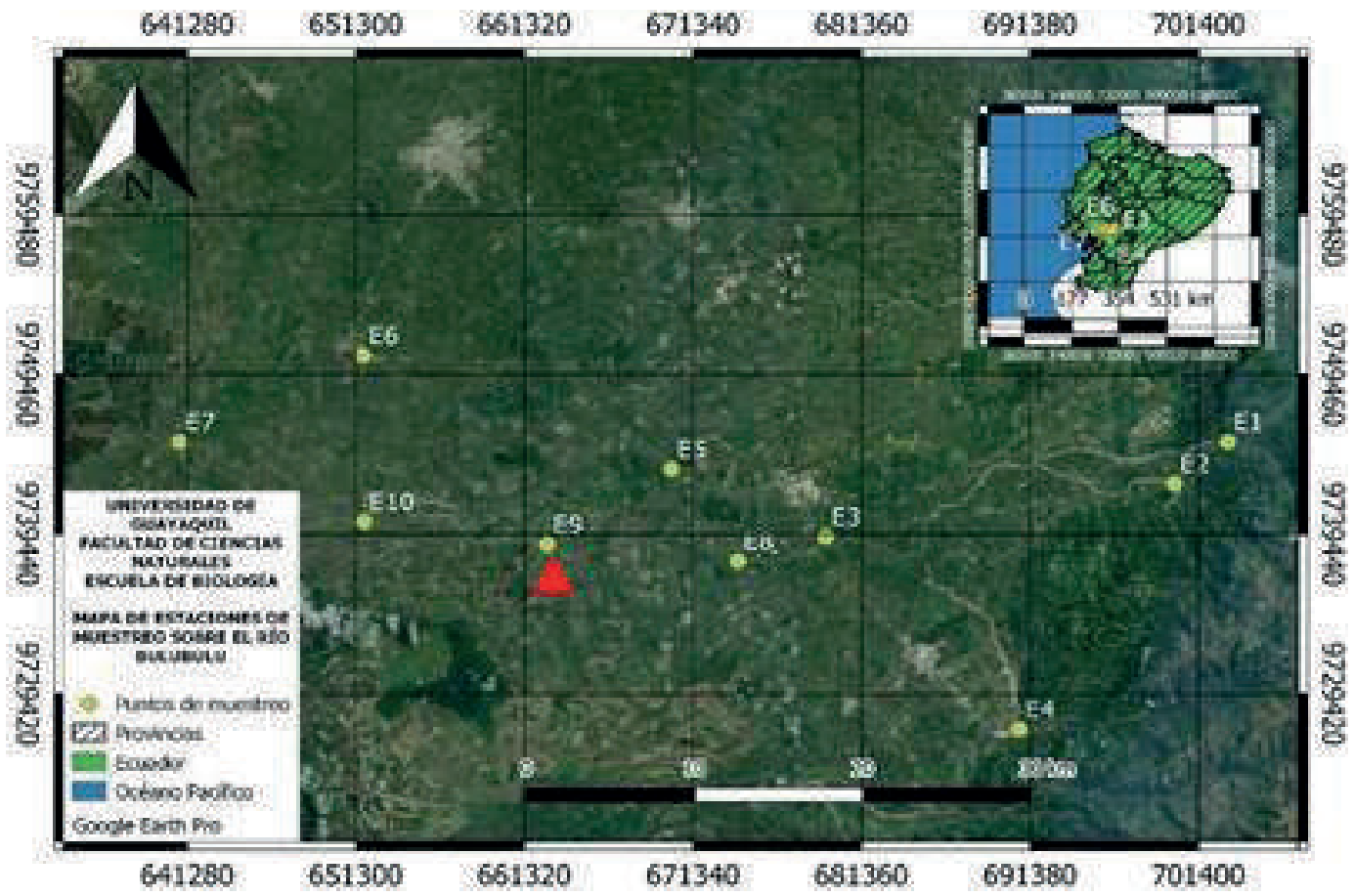

Figura 5. Estaciones de muestreo que presentaron especímenes de Achirus scutum en el río Bulubulu 
Rev. Cient. Cien. Nat. Ambien. 15(1):211-227 Junio 2021
Núñez \& Torres - Diversidad, abundancia y distribución de peces en el río Bulubulu

Tabla 8. Composición y estructura de la ictiofauna presente en la estación 6 .

\begin{tabular}{ccccc}
\hline & \multicolumn{5}{c}{ Estación 6 } & & Porcentaje \% \\
\hline Orden & Familia & N. científico & N. de individuos & 15,4 \\
Characiformes & Bryconidae & Brycon alburnus & 8 & 3,8 \\
Characiformes & Characidae & Rhoadsia altipinna & 2 & 11,5 \\
Perciformes & Cichilidae & Andinoacara rivulatus & 6 & 36,5 \\
Characiformes & Characidae & Astyanax festae & 19 & 32,7 \\
Characiformes & Bryconidae & Brycon dentex & 17 & 100,0 \\
\hline
\end{tabular}

Tabla 9. Composición y estructura de la ictiofauna presente en la estación 7.

\begin{tabular}{ccccc}
\hline \multicolumn{5}{c}{ Estación 7 } \\
\hline Orden & Familia & N. científico & N. de individuos & Porcentaje \% \\
\hline Characiformes & Bryconidae & Brycon alburnus & 19 & 36,5 \\
Characiformes & Characidae & Astyanax festae & 10 & 19,2 \\
Characiformes & Bryconidae & Brycon dentex & 23 & 44,2 \\
\hline & TOTAL & & $\mathbf{5 2}$ & $\mathbf{1 0 0 , 0}$ \\
\hline
\end{tabular}

Tabla 10. Composición y estructura de la ictiofauna presente en la estación 8.

\begin{tabular}{ccccc}
\hline \multicolumn{5}{c}{ estación 8 } \\
\hline orden & Familia & N. científico & N. de individuos & Porcentaje \% \\
\hline Characiformes & Characidae & Astyanax festae & 10 & 40,0 \\
Characiformes & Bryconidae & Brycon dentex & 2 & 8,0 \\
Perciformes & Cichilidae & Oreochromis sp. & 5 & 20,0 \\
Characiformes & Bryconidae & Brycon alburnus & 8 & 32,0 \\
\hline & TOTAL & & $\mathbf{2 5}$ & 100,0 \\
\hline
\end{tabular}

Tabla 11. Composición y estructura de la ictiofauna presente en la estación 9.

\begin{tabular}{ccccc}
\hline & \multicolumn{5}{c}{ Estación 9} \\
\hline orden & Familia & N. científico & N. de individuos & Porcentaje \% \\
\hline Characiformes & Characidae & Astyanax festae & 11 & 28,9 \\
Characiformes & Bryconidae & Brycon dentex & 6 & 15,8 \\
Perciformes & Cichilidae & Oreochromis sp. & 14 & 36,8 \\
Pleuronectiformes & Achiridae & Achirus scutum & 3 & 7,9 \\
Siluriformes & Heptateridae & Pimelodella modestus & 4 & 10,5 \\
\hline & TOTAL & & 38 & 100,0 \\
\hline
\end{tabular}

Tabla 12. Composición y estructura de la ictiofauna presente en la estación 10.

\begin{tabular}{clccc}
\hline & & Estación 10 & \\
\hline Orden & Familia & N. científico & $\begin{array}{c}\text { N. de } \\
\text { individuos }\end{array}$ & Porcentaje \% \\
\hline Cyprinodontiformes & Poeciliidae & Poecilia reticulata & 6 & 16,7 \\
Characiformes & Bryconidae & Brycon dentex & 9 & 25,0 \\
Perciformes & Cichilidae & Oreochromis sp. & 16 & 44,4 \\
Characiformes & Characidae & Eretmobrycon brevirostris & 5 & 13,9 \\
& TOTAL & & 36 & 100,0 \\
\hline
\end{tabular}




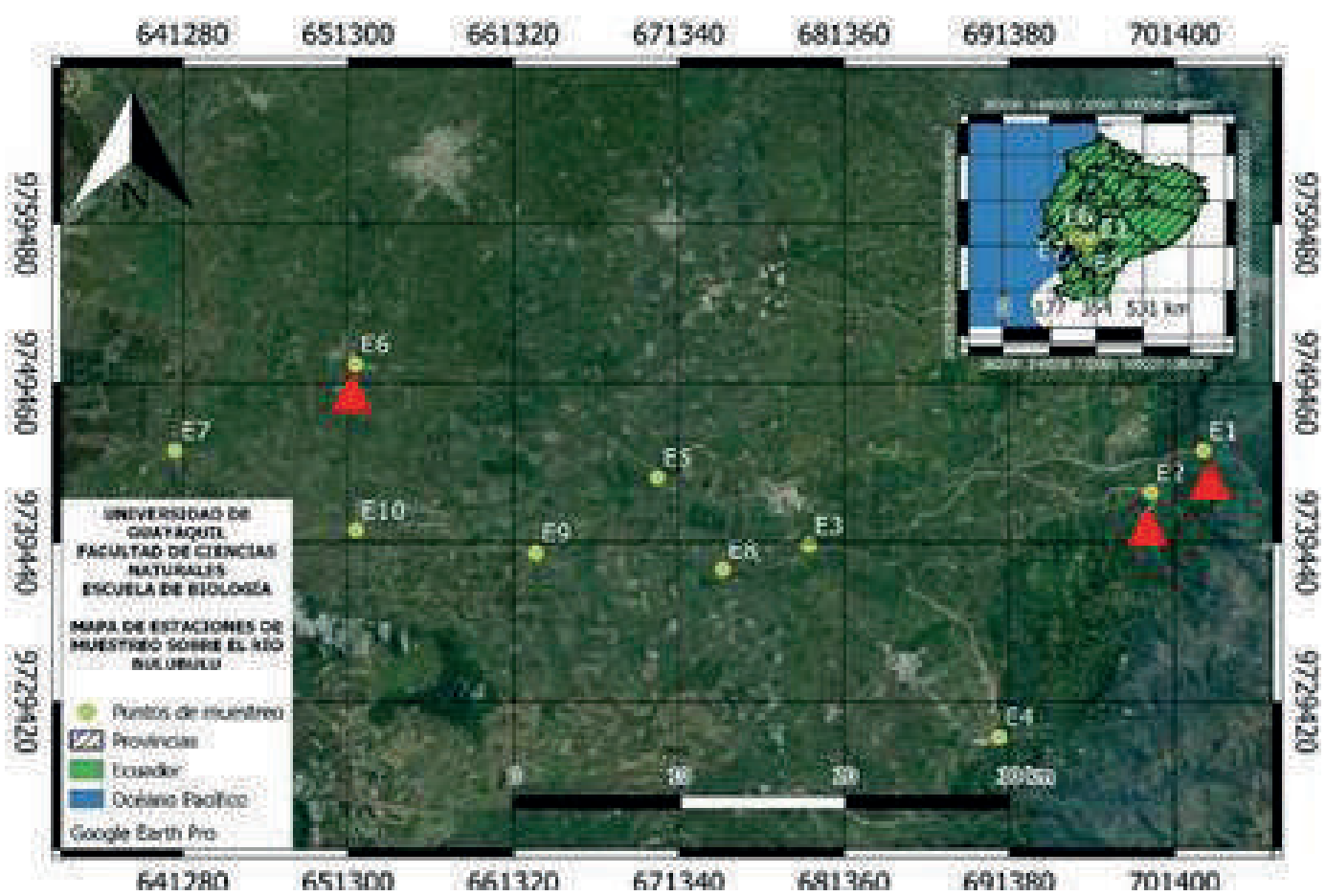

Figura 6. Estaciones de muestreo que presentaron especímenes de Andinoacara rivulatus en el río Bulubulu.

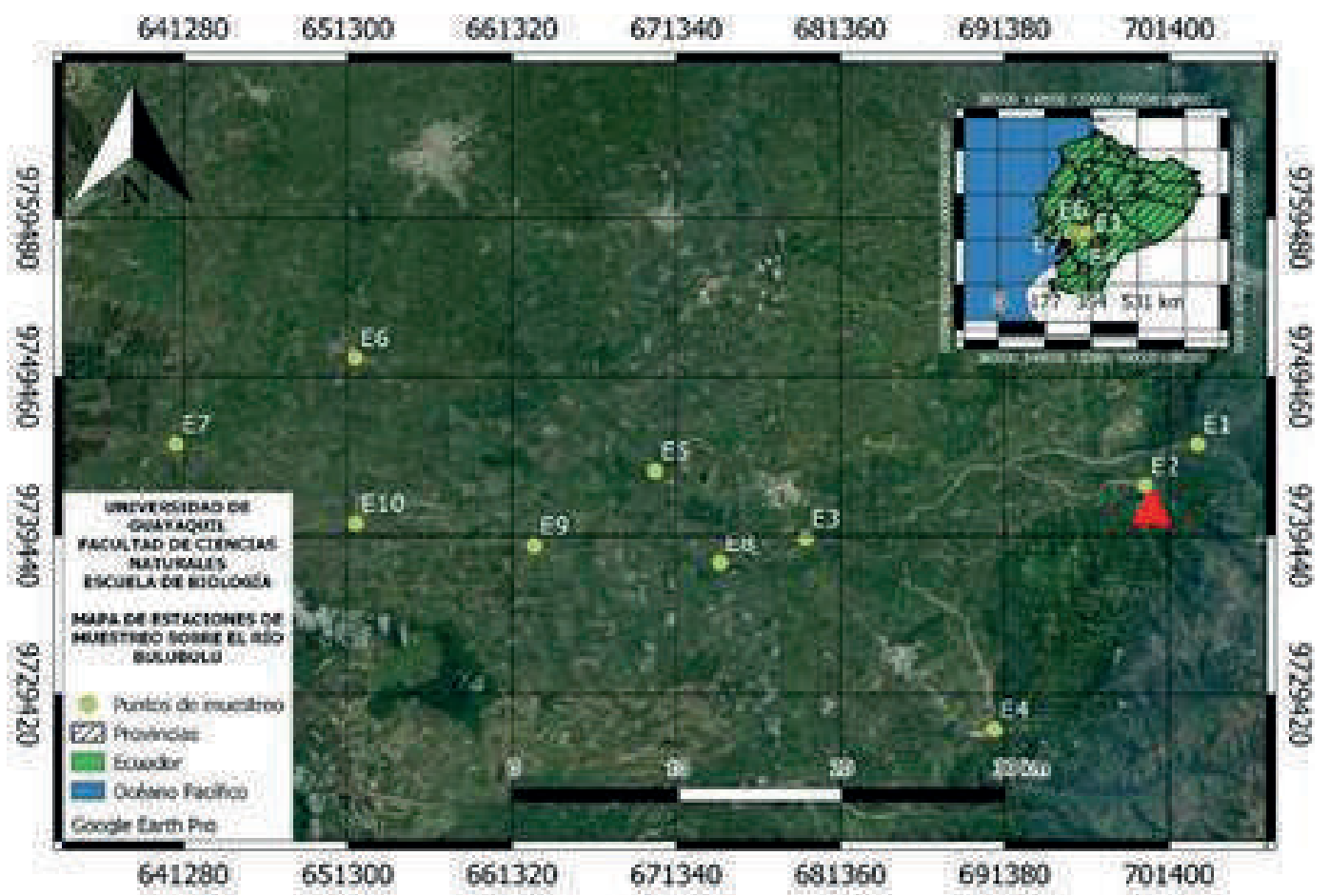

Figura 7. Estaciones de muestreo que presentaron especímenes de Astroblepus sp. en el río Bulubulu 
Rev. Cient. Cien. Nat. Ambien. 15(1):211-227 Junio 2021
Núñez \& Torres - Diversidad, abundancia y distribución de peces en el río Bulubulu

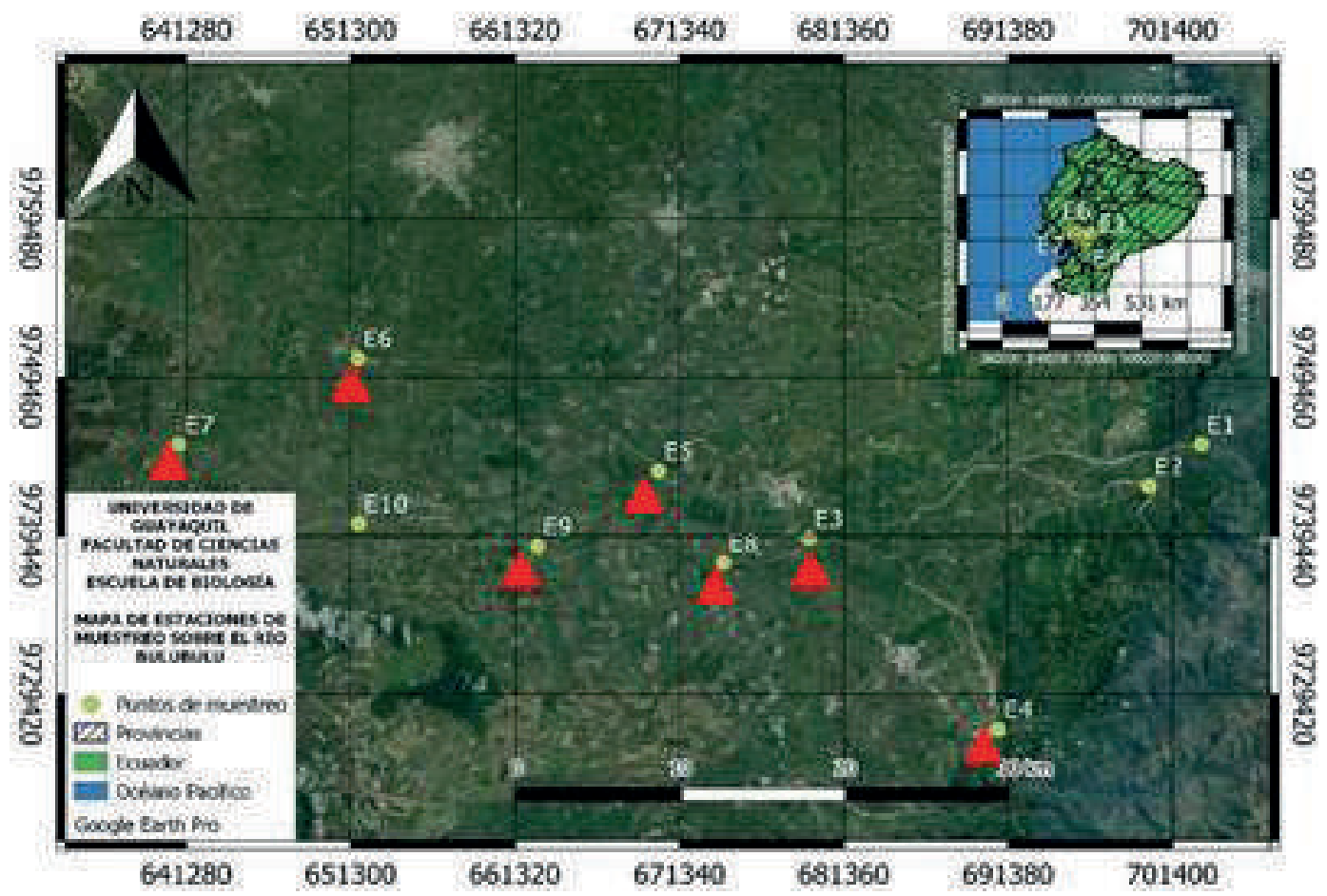

Figura 8. Estaciones de muestreo que presentaron especímenes de Astyanax festae en el río Bulubulu

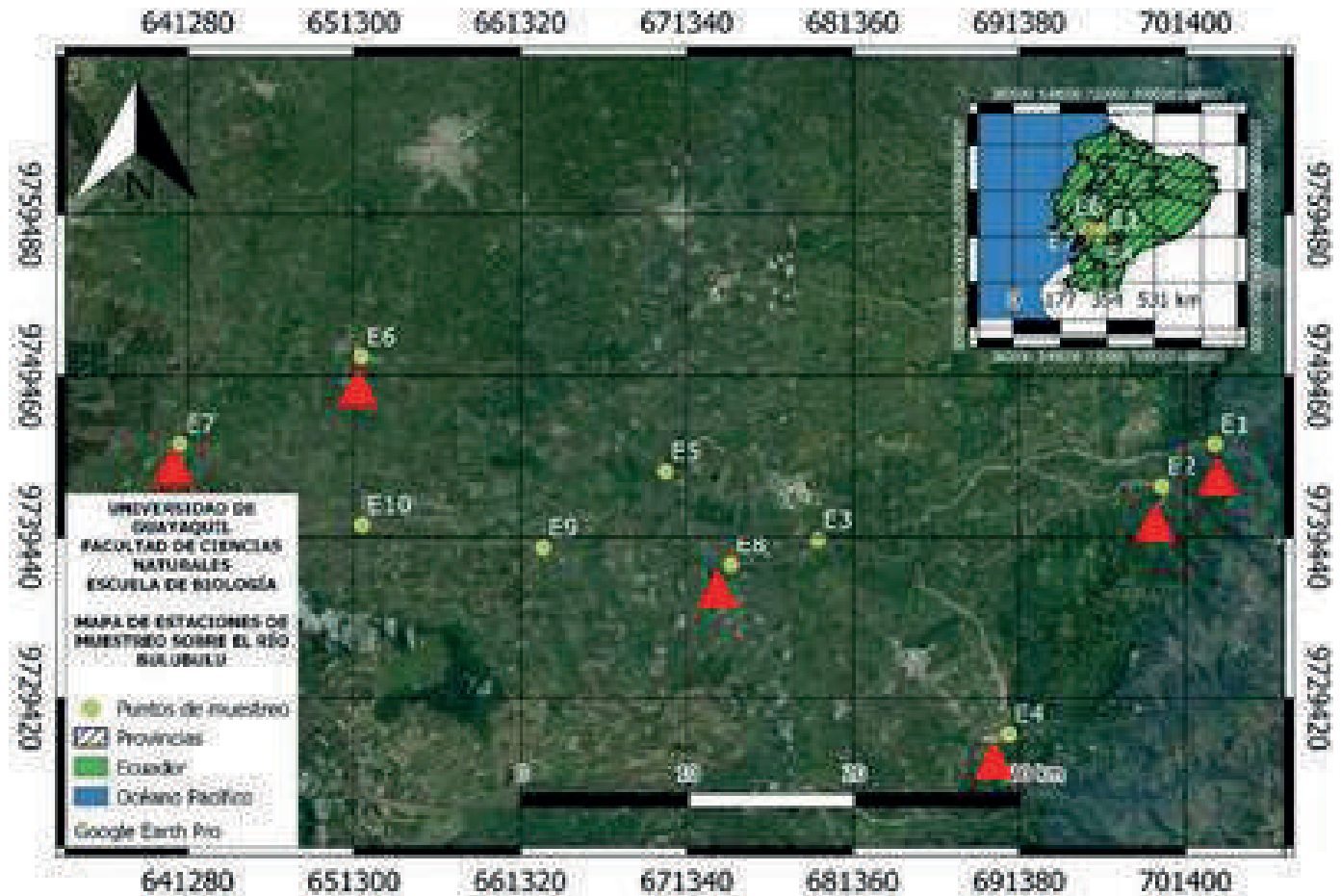

Figura 9. Estaciones de muestreo que presentaron especímenes de Brycon alburnus en el rio Bulubulu 


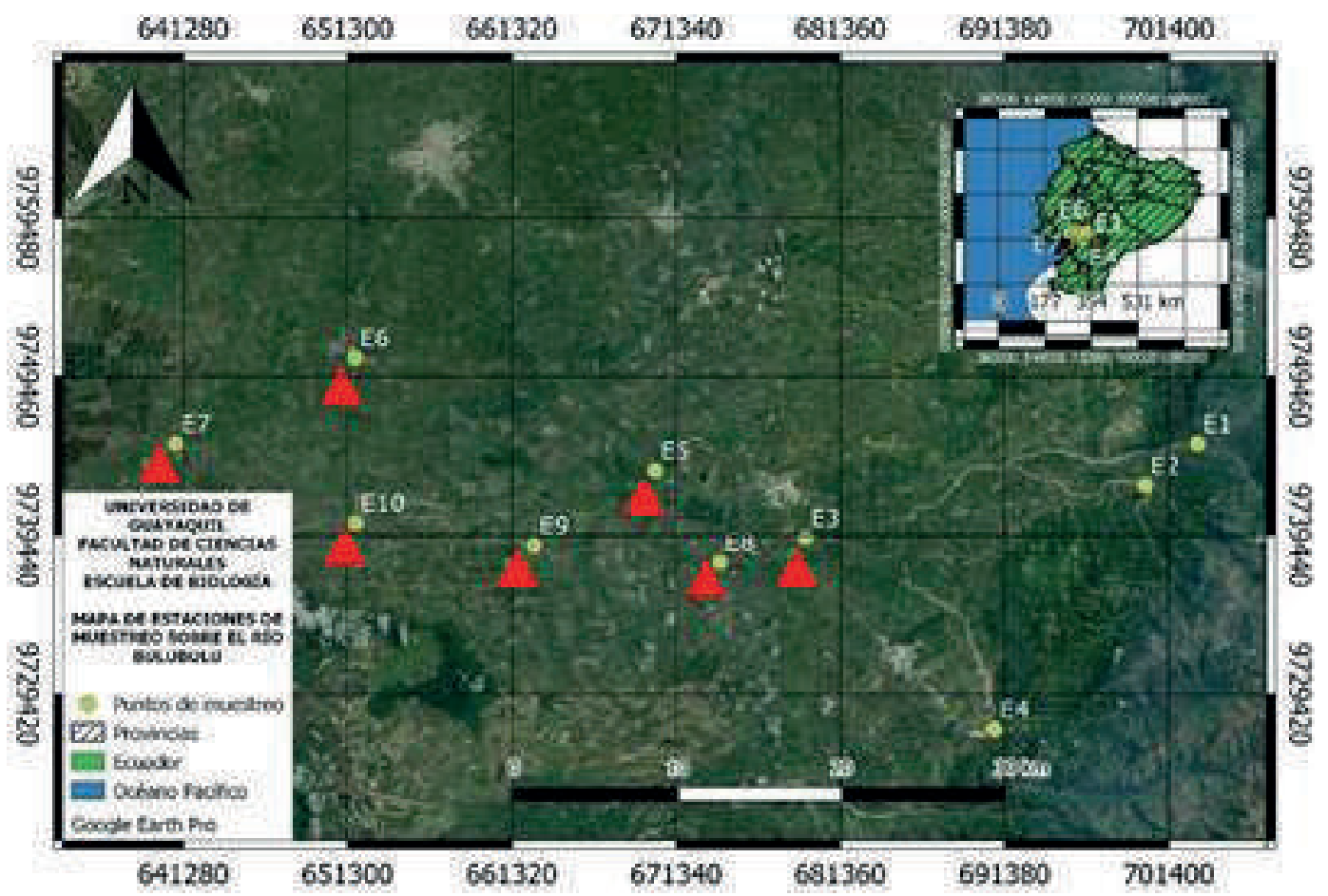

Figura 10. Estaciones de muestreo que presentaron especímenes de Brycon dentex en el rio Bulubulu

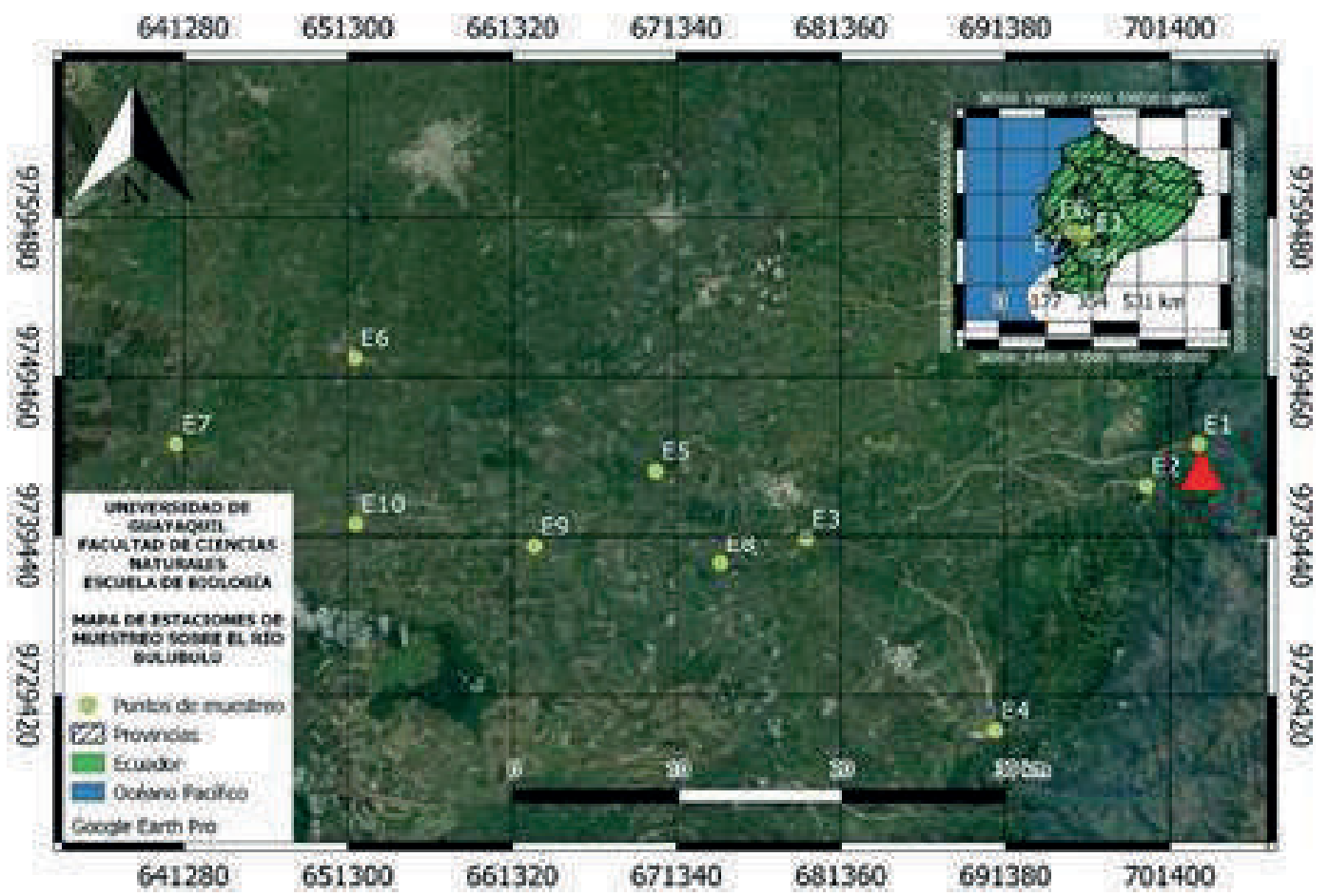

Figura 11. Estaciones de muestreo que presentaron especímenes de Chaetostoma fischeri en el rio Bulubulu 
Rev. Cient. Cien. Nat. Ambien. 15(1):211-227 Junio 2021
Núñez \& Torres - Diversidad, abundancia y distribución de peces en el río Bulubulu

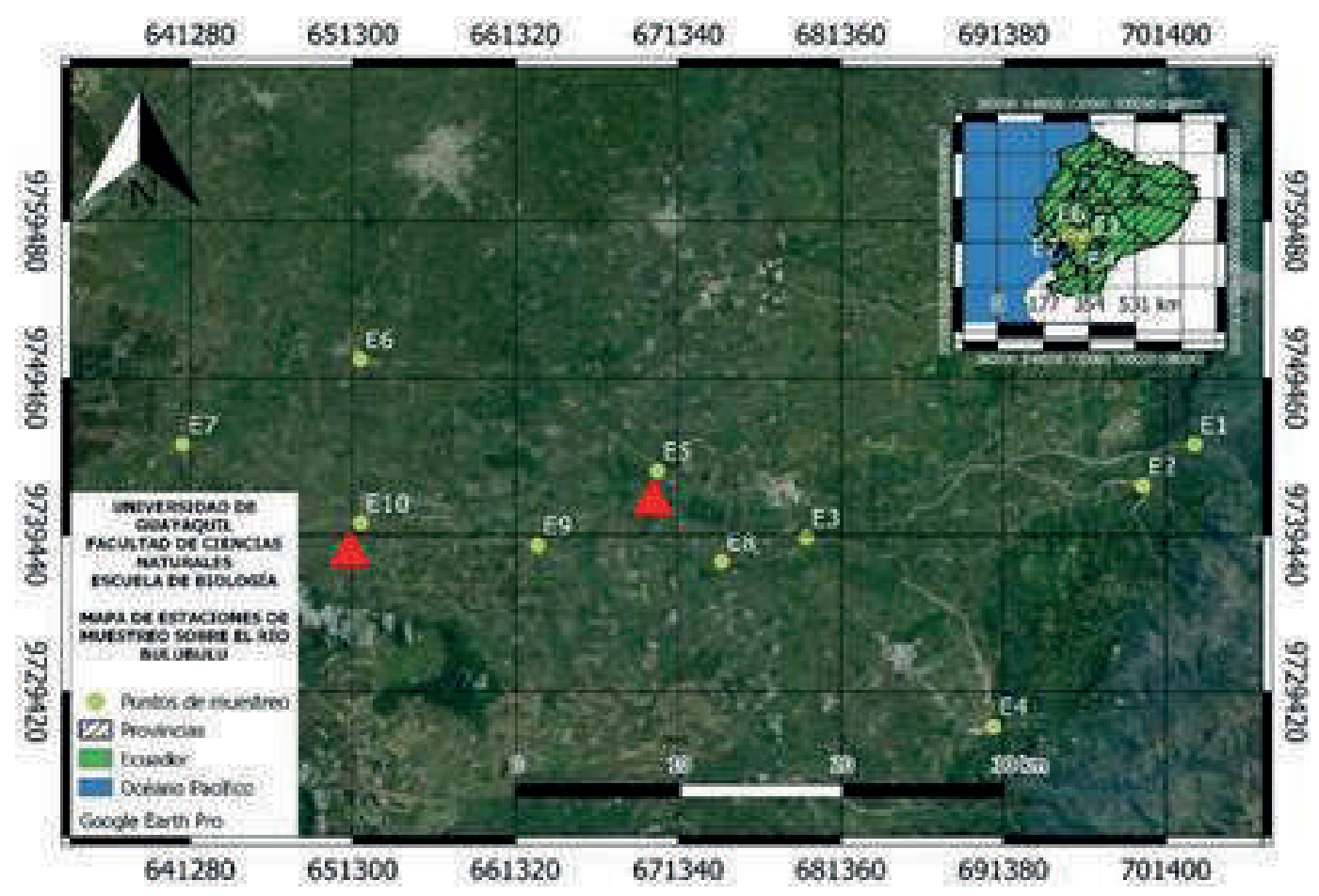

Figura 12. Estaciones de muestreo que presentaron especímenes de Eretmobrycon brevirostris en el rio Bulubulu

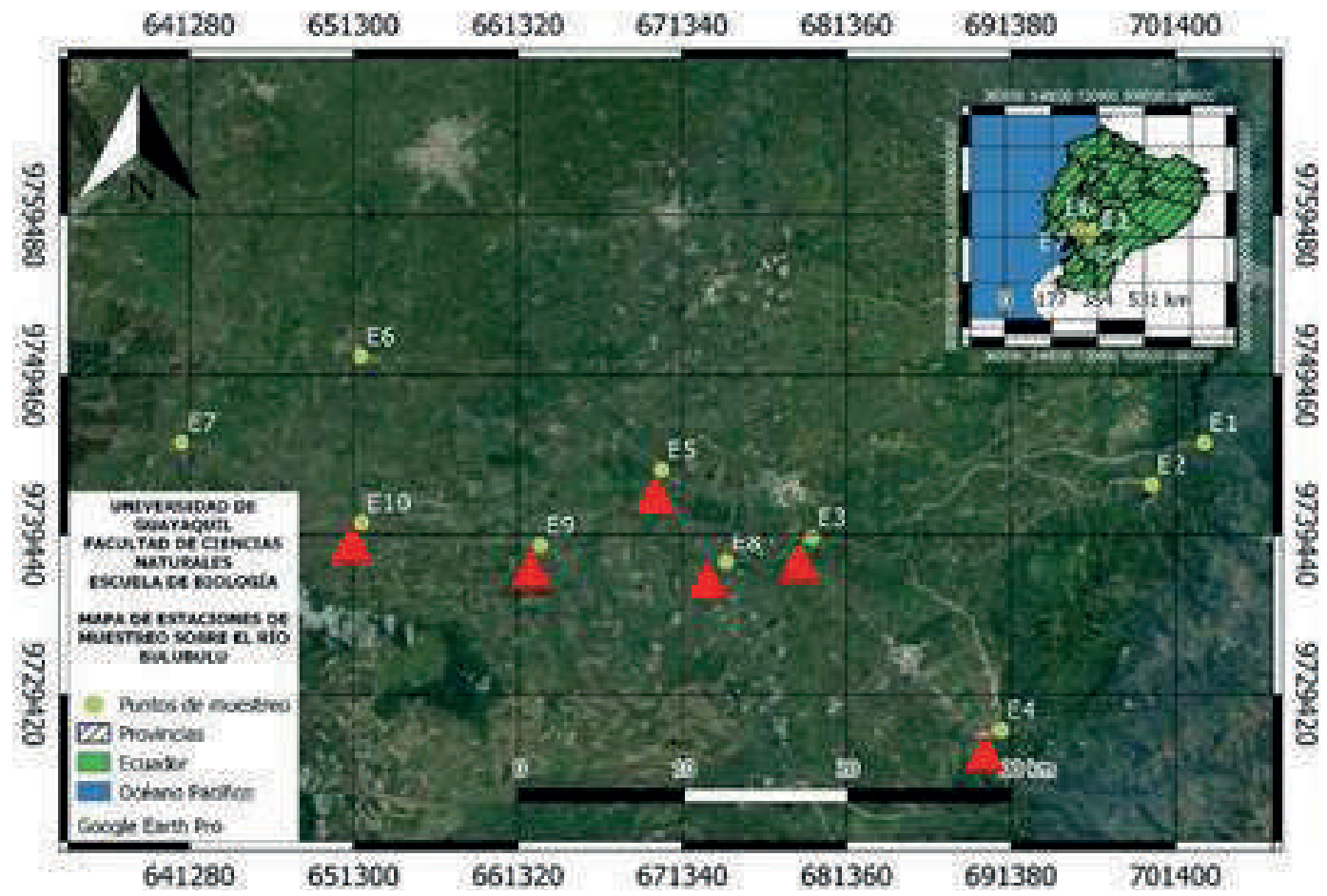

Figura 13. Estaciones de muestreo que presentaron especímenes de Oreochromis sp. en el rio Bulubulu 


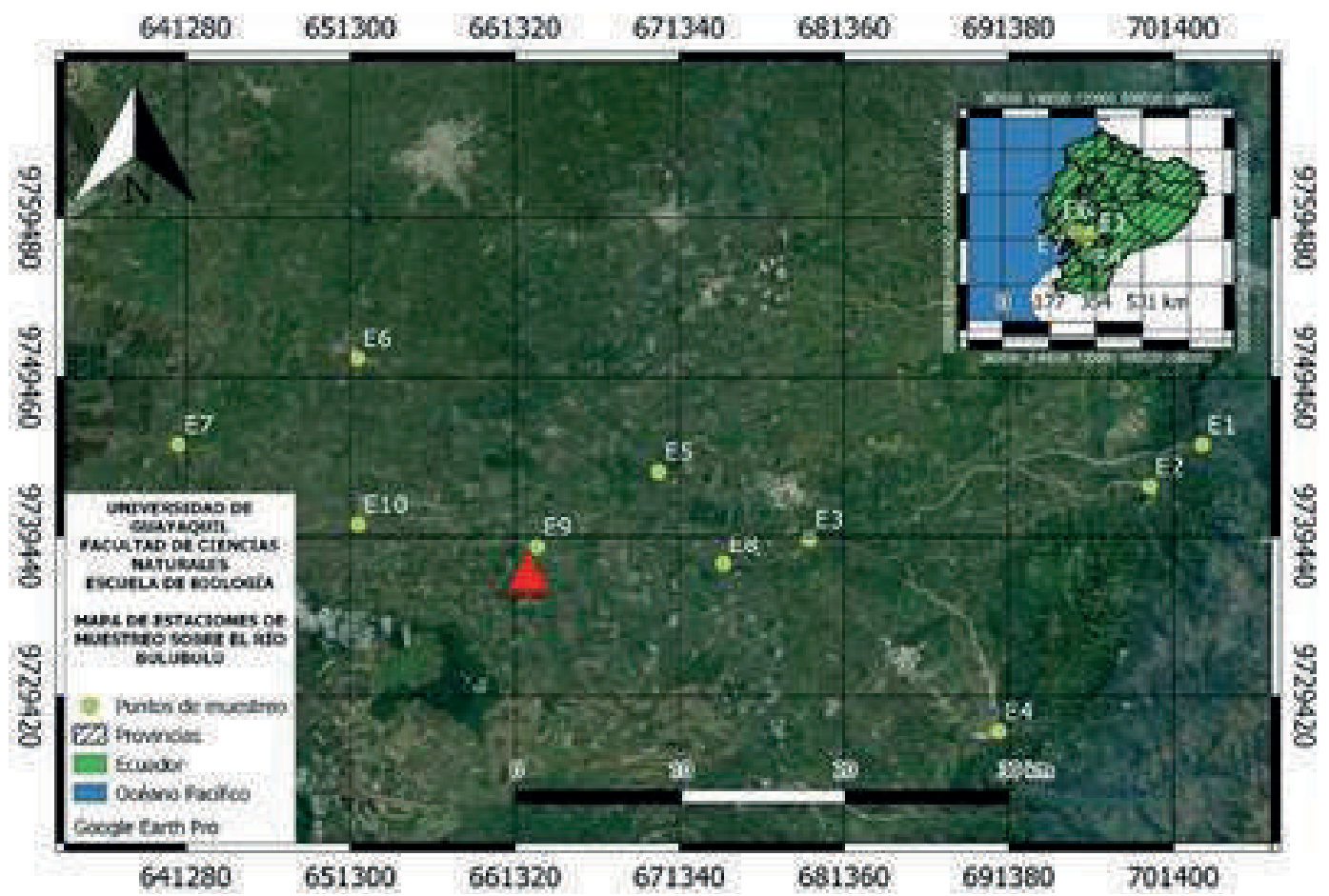

Figura 14. Estaciones de muestreo que presentaron especímenes de Pimelodella modestus en el rio Bulubulu.

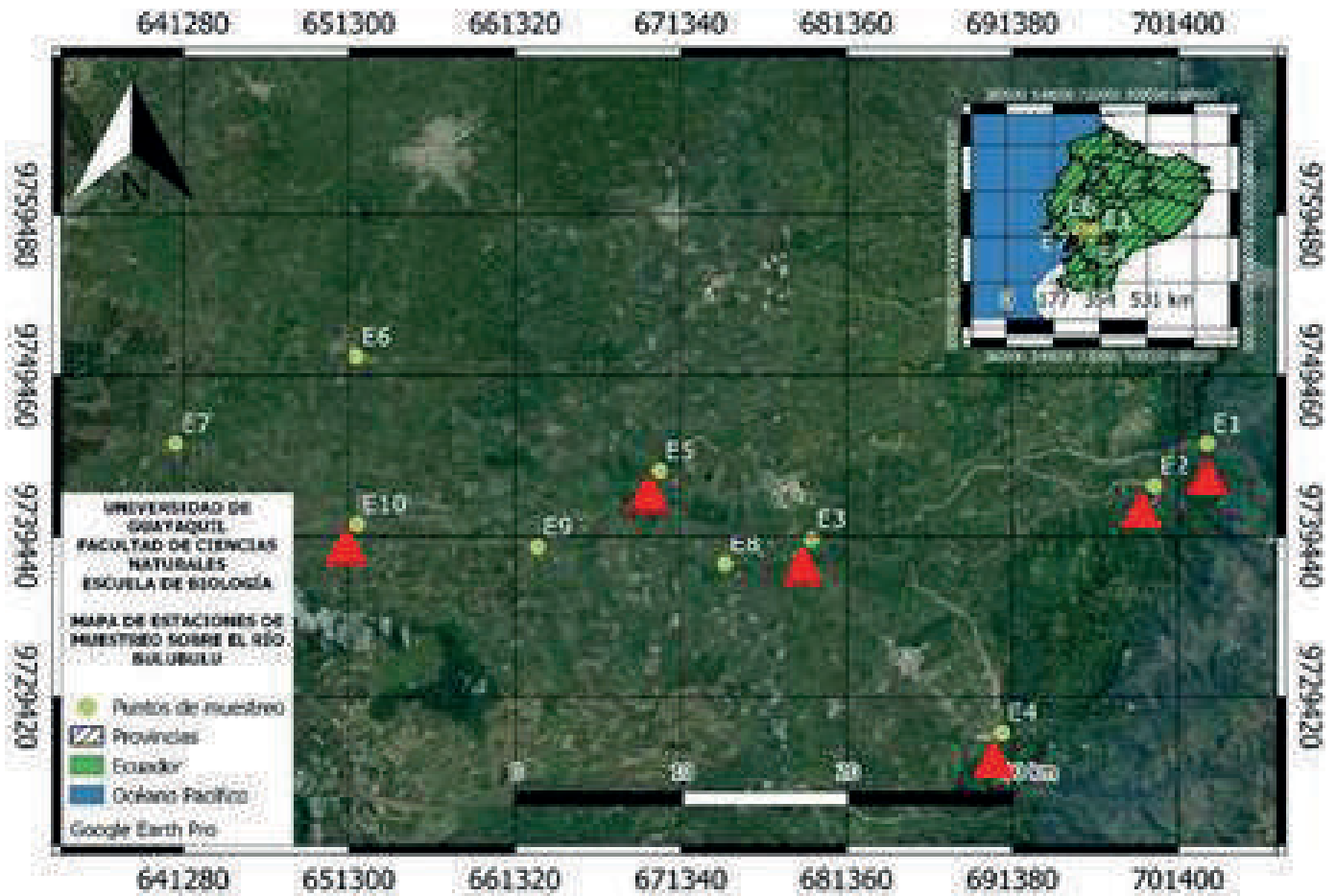

Figura 15. Estaciones de muestreo que presentaron especímenes de Poecilia reticulata en el rio Bulubulu 


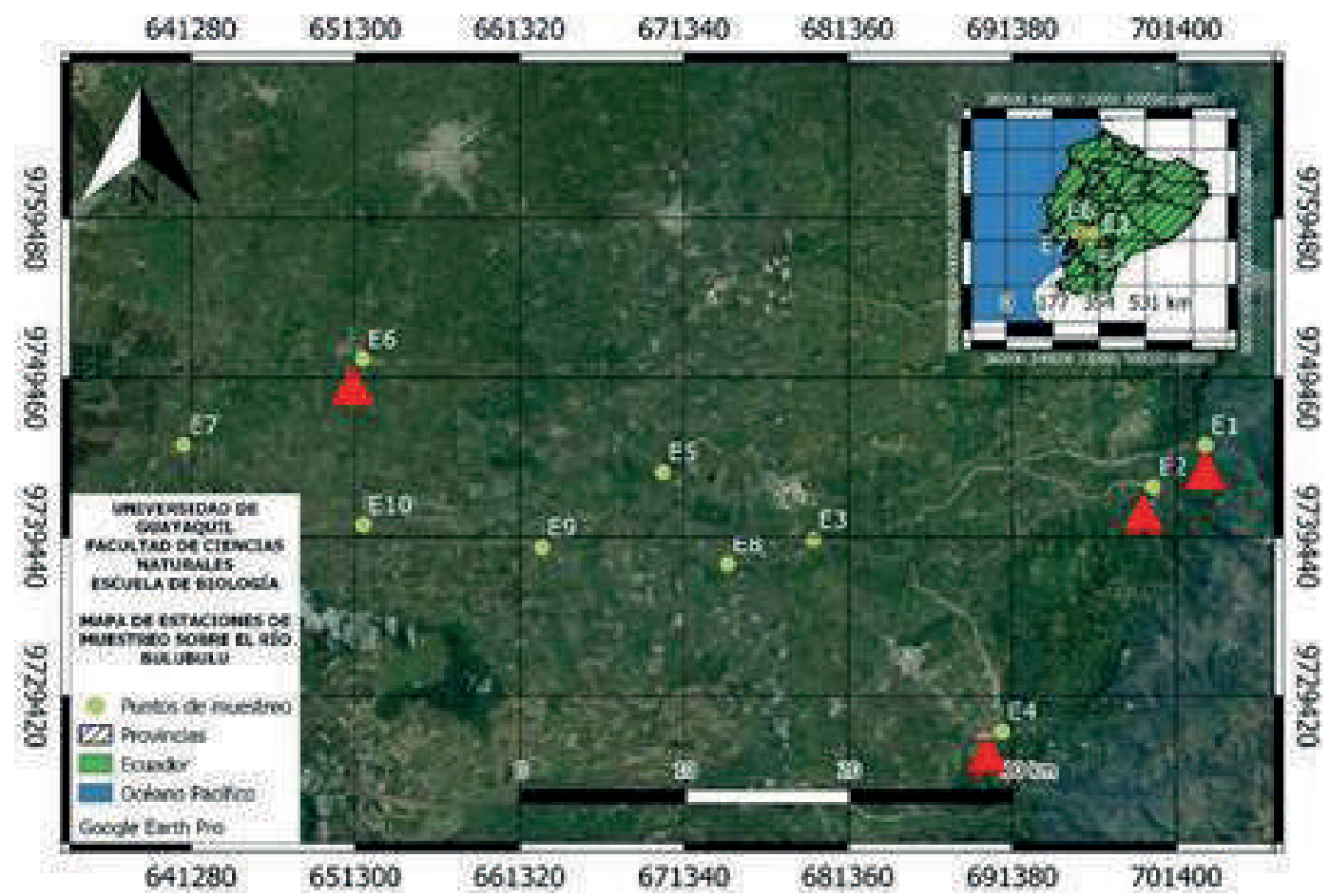

Figura 16. Estaciones de muestreo que presentaron especímenes de Rhoadsia altipinna en el rio Bulubulu

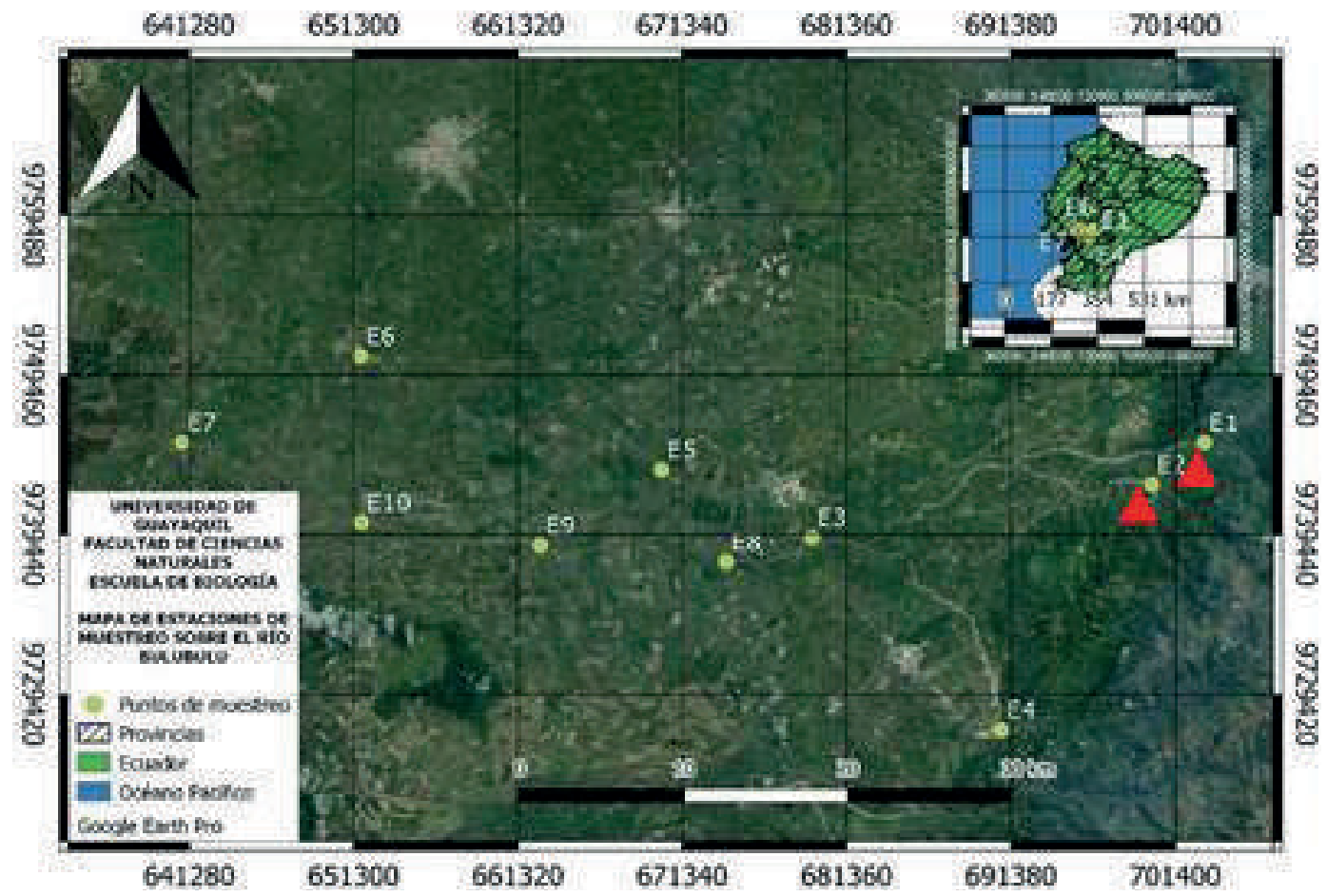

Figura 17. Estaciones demuestreo que presentaron especímenes de Transancistrus santarosensis en el rio Bulubulu 


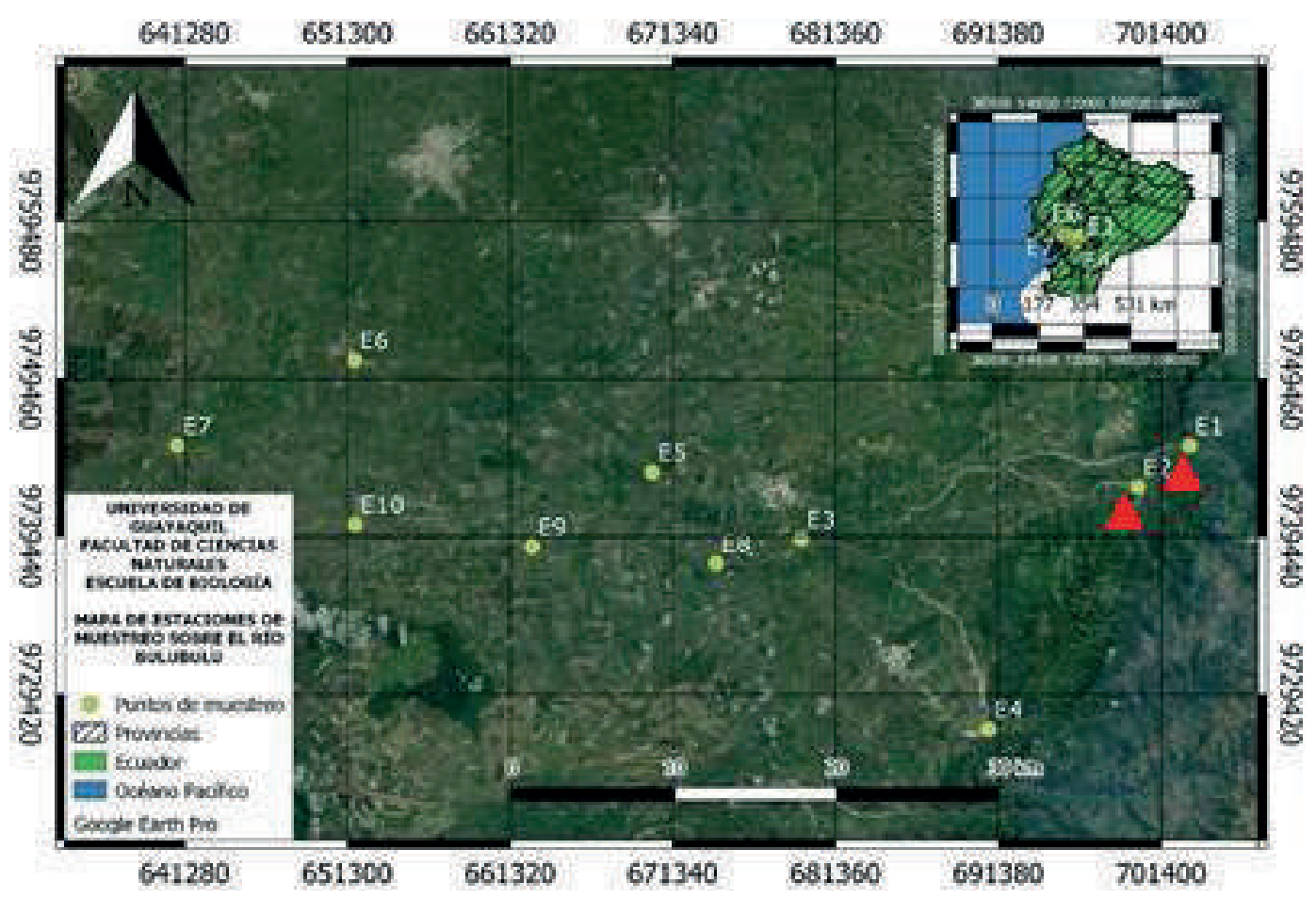

Figura 18. Estaciones demuestreo que presentaron especímenes de Trychomicterus taenia en el rio Bulubulu

\section{Discusión}

En este estudio se identificaron un total de 14 especies de peces. Aquello representa el 1,6\% de la ictiofauna de agua dulce del Ecuador según lo reportado por Barriga (2012), quien identificó 944 especies. Stewart et al. (1987) identificó alrededor de 473 especies en el rio Napo, lo cual se relaciona con la extensión de este rio, sus afluentes y la ubicación geográfica del mismo (selva amazónica).

Nuestros resultados se asemejan a los reportados por Aucapiña (2017) en Rio Ruidoso y Mawyin (2017) en el Rio Culebra, quienes encontraron 19 y 9 especies de peces, respectivamente. Sin embargo, en el estudio realizado en Rio Bulubulu se obtuvo un total de 428 individuos, un resultado similar al encontrado en el rio Culebra (437 individuos), pero diferenciándose en gran manera del trabajo realizado por Aucapiña, el cual presentó un total de 1965 individuos capturados. Esto se debería a que el rio estudiado por ese autor atraviesa una reserva (Manglares Churute), por lo tanto, existe una mayor conservación de la ictiofauna en esa cuenca.

Massay (2009) en su estudio de la cuenca baja del rio Taura, parte de la cuenca del rio Bulubulu, identificó 25 especies. Este es un valor mayor al obtenido en el presente estudio, que podría relacionarse con la diferencia temporal y con la influencia antropogénica en esta parte del rio.
Mientras en el estudio hecho por Massay se lograron identificar cuatro nuevas especies, durante la realización del presente trabajo no se pudo encontrar nuevos registros y se obtuvo una baja diversidad. Aquello podría deberse a la diferencia estacional en el estudio, ya que en temporada lluviosa el índice de abundancia es mayor.

En cuanto a la abundancia relativa, se comparte el resultado al obtenido por Aucapiña (2017) el cual determinó que Astyanax festae con 610 individuos era la especie más abundante en el área de estudio, esto podría ser resultado de las características físicas similares entre ambos ríos. Sin embargo, esta conclusión no es compartida por el estudio realizado en el rio Culebra, el cual menciona a Oreochromis sp. como la especie más abundante, con un total de 81 individuos,

esta diferencia se puede explicar observando los puntos de muestreo (seis), un número inferior al utilizado en el presente estudio.

Se observa una gran diferencia en riqueza de especies en comparación con el trabajo realizado por Laaz \& Torres (2015) en la cuenca del río Guayas, donde se obtuvo un total de 125 especies, de las cuales seis eran introducidas. En el presente estudio solo se encontró una especie de este tipo (Oreochromis sp.), esto debido a la diferencia en morfología y extensión de las cuencas estudiadas, además de existir grandes 
diferencias en cuanto a taxonomía, debido a que en el primer trabajo se cita a la especie Bryconamericus brevirostris, la cual cambio su taxonomía recientemente, ya que en el estudio realizado por Thomaz, Arcila, Ortí y Malabarba (2015). Se lo trasladó al género Eretmobrycon. Esto refleja la importancia de realizar estudios constantemente, para actualizar listas de especies y registrar cambios que pueden suscitarse debido a la actualización taxonómica.

Las especies Brycon alburnus y Brycon dentex formaban parte de la familia Characidae, denominación que cambió gracias al trabajo realizado por Oliveira et al. (2015). Ahora formando parte de la familia Bryconidae. Por esta razón, en trabajos anteriores se aprecia esta diferencia en la taxonomía de ambas especies, sin embargo, en este trabajo se realizaron las identificaciones taxonómicas bajo esta nueva clasificación.

\section{Conclusiones}

En el presente estudio realizado en el rio Bulubulu la abundancia total fue de 428 individuos. De los cuales solamente una especie es introducida (Oreochromis sp.). La cual tuvo una abundancia relativa del 15,9\% y una distribución media.

El orden más abundante fue Characiformes y el orden Siluriformes fue el más diverso

En el orden Characiformes, la familia más abundante fue Bryconidae y la más diversa fue Characidae

A nivel de especie, las que obtuvieron una mayor representatividad fueron Astyanax festae y Brycon dentex, ambas comprenden el $42 \%$ del total de individuos muestreados.

Astyanax festae y Brycon dentex tuvieron una distribución más amplia, compartiendo hábitat en seis estaciones.

\section{Recomendaciones}

- Se recomienda realizar estudios estacionales en este río para analizar la fluctuación en la diversidad y abundancia del mismo.

- $\quad$ Realizar muestreos relacionados al tipo de cuenca (alta, media y baja), debido a los cambios que podrían ocurrir a nivel diversidad de especies

- Realizar un estudio sobre la afectación antropogénica en la cuenca baja del río Bulubulu, a nivel de calidad de agua e ictiofauna.

- Efectuar investigaciones sobre la biología de las especies presentes en el río Bulubulu, debido a los cambios que podrían estar suscitándose por las afectaciones antropogénicas en diferentes partes de la cuenca del río.

\section{Bibliografía}

Anaguano, F. (2013). Peces de la Laguna Cormorán, Parque Nacional Sangay, Ecuador. Universidad Central del Ecuador, Escuela de Ciencias Biológicas y Ambientales. Pág, 9.

Anzueto, M., Velázquez, E., Gómez, A., Quiñonez, R., y Olson, B. (2013). Peces de la reserva de la biosfera selva El Ocote, Chiapas, México. Universidad de ciencias y artes de Chiapas. Pág, 139.

Aucapiña, C. (2017). Inventario ictiológico del Río Ruidoso ("recinto El Mango" - provincia del Guayas). Universidad de Guayaquil, Facultad de Ciencias Naturales.

Barriga, R. (1991). Lista de peces de agua dulce e intermareales de Ecuador.

Escuela Politecnica Nacional. Quito.

Barriga, R. (1994). Peces del noroeste del Ecuador. Politécnica 19(2):43-154.

Recuperado de http://bibdigital.epn.edu.ec/ handle/15000/5084.

Barriga, R. (2012). Lista de peces de agua dulce e intermareales del Ecuador.

Escuela Politenica Nacional. Quito.

Böhlke, J. (1958). Studies on Fishes of the family Characidae. $\mathrm{N}^{\circ}$ 14. A report on several extensive recent collections from Ecuador. Proceedings of the Academy of Natural Sciences. Philadelphia Pp: 110:1-121.

Vecinos de Pedro J. Montero denuncian contaminación del río Bulubulu (20 de diciembre d e l 2016). El telégrafo. Recuperadode: https:// www.eltelegrafo.com.ec/noticias/ecuador/1/vecinosde-pedro-j- montero-denuncian-contaminacion-del-riobulubulu

Esta noticia ha sido publicada originalmente por Diario EL TELÉGRAFO bajo la siguiente dirección:

https://www.eltelegrafo.com.ec/noticias/ecuador/1/ vecinos-de-pedro-j- montero-denuncian-contaminaciondel-rio-bulubulu

Si va a hacer uso de la misma, por favor, cite nuestra fuente y coloque un enlace hacia la nota original. www.eltelegrafo. com.ec

Helfman, C., Collette. B., y Facey, D. (1997). The diversity of fishes. Blackwell Science. USA. 528 pp.

Jiménez, L., Álvarez, J., Ochoa, L., Loaiza, A., Londoño, J., Restrepo, D., Aguirre, K., Hernández, A., Correa J., y Jaramillo-Villa U. (2014). Guía Ilustrada Peces Cañón del río Porce, Antioquia. EPM. Universidad de Antioquia, Herbario Universidad de Antioquia - Medellín, Colombia. Pág, 106.

Laaz, E., y Torres, A. (2014). "Lista de peces continentales de la Cuenca del Río Guayas". Guía FAO para la identificación de especies para los fines de la pesca Pacifico-Oriental. Pág, 45

Leveque, C., Oberdorff, T., Paugy D., Stiassny M., Tedesco, P. (2008). Global diversity of fish (Pisces) in freshwater. Hydrobiologia (2008). 595:545-567 pp.

López, A., Gallegos, M., Gómez, J., Rodríguez, J., Ledesma, L., Rodríguez, J., y Angón, E. (2015). Adaptación y curvas de crecimiento de la vieja azul (Andinoacara rivulatus) y vieja colorada (Cichlasoma festae), cultivadas bajo condiciones controladas. Universidad Técnica de Babahoyo. Pág, 110.

Maldonado, O., Ortega, J., Usma O., Galvis V., Villa-Navarro, F., Vásquez G., Prada- Pedreros, S., y Ardila R. (2005). Peces de los Andes de Colombia. Instituto de Investigación de Recursos Biológicos «Alexander von Humboldt». Bogotá, D.C. Colombia. Pág, 346.

Massay, S. (2009). Peces estuarinos y dulceacuicolas de la cuenca baja del rìo Taura. Universidad de Guayaquil. 
Ecuador.

Mawyin, A. (2017). Diversidad y abundancia ictiofaunística en el río Culebra (Guayas - Ecuador). Universidad de Guayaquil, Facultad de Ciencias Naturales.

Nelson, J.S. (2006). Fishes of the World. 4th Edition. John Wiley y Sons, Hoboken, New Jersey, 624 pp.

Oliveira, C., Avelino, G.S., Abe, K.T., Mariguela, T. C., Benine, R. C., Orti, G., Vari, R. P., y Correa e Castro, R. M. (2011). Phylogenetic relationships

within the speciose family Characidae (Teleostei: Ostariophysi: Characiformes) based on multilocus analysis and extensive ingroup sampling. BMC Evol Biol 11, 275.

Ovchynnyk, M. (1967). Freshwater fishes of Ecuador, Latina America Studies Center. Michigan State University. Pp: 1-44.

Ovchynnyk, M. (1968). Annotated list of the frshwater fish of Ecuador.

Zoologistcher Anzeiger. Pp: 237-268.

Ovchynnyk, M. (1971). Peces de agua dulce del Ecuador y sus perspectivas para desarrollar su cultivo. Latin American Studies Center. Michigan State University, USA. Pp: 142.

Reis, E., Kullander, O., y Ferraris, Jr. (2003). Check list of the freshwater fishes of South and Central America. Edipucrs, Sao Paulo. Pág, 120.

Stewart, D., Barriga, R. \& Ibarra, M. (1987). Ictiofauna de la cuenca del río Napo.

Thomaz, A., Arcila, D., Ortí, G., \& Malabarba, L. (2015). Molecular phylogeny of the subfamily Stevardiinae Gill, 1858 (Characiformes: Characidae): classification and the evolution of reproductive traits. BMC Evolutionary Biologyvolume, 15(146).

Tognelli, M., Lasso, C., Bota-Sierra, C., Jiménez-Segura, L. y Cox, N. (2016). Estado de Conservación y Distribución de la Biodiversidad de Agua Dulce en los Andes Tropicales. Gland, Suiza, Cambridge, UK y Arlington, USA: UICN. 199 pp.

Varela, L. A., Ron, S. R. 2018. Geografía y clima del Ecuador. BIOWEB. Pontificia Universidad Católica del Ecuador. Disponible en

<https://bioweb.bio/geoclima/> Consulta: 31 de enero 2019. 